\title{
Friction Stir Processing on Carbon Steel
}

\author{
Sergei Yu. Tarasov ${ }^{1,2, a), ~ A l e x a n d e r ~ G . ~ M e l n i k o v, ~}{ }^{2, \text { b) }}$ \\ and Valery E. Rubtsov, ${ }^{1, c)}$ \\ ${ }^{1}$ Institute of Strength Physics and Materials Science SB RAS, Tomsk, 634055, Russia \\ ${ }^{2}$ National Research Tomsk Polytechnic University, Tomsk, 634050, Russia \\ a) Corresponding author: tsy@ispms.ru \\ b) melnikov_ag@tpu.ru \\ c) rvy@ispms.ru
}

\begin{abstract}
Friction stir processing of medium carbon steel samples has been carried out using a milling machine and tools made of cemented tungsten carbide. Samples have been machined from 40 and $40 \mathrm{X}$ steels. The tools have been made in the shape of $5 \times 5 \times 1.5 \mathrm{~mm}$ and $3 \times 3 \times 1.5 \mathrm{~mm}$ tetrahedrons. The microstructure of stirred zone has been obtained using the smaller tool and consists of fine recrystallized 2-3 $\mu \mathrm{m}$ grains, whereas the larger tool has produced the "onion-like" structures comprising hard quenched "white" 500-600 MPa layers with 300-350 MPa interlayers of bainite needles. The mean values of wear intensity obtained after measuring the wear scar width were $0.02 \mathrm{~mm} / \mathrm{m}$ and $0.001 \mathrm{~mm} / \mathrm{m}$ for nonprocessed and processed samples, respectively.
\end{abstract}

Keywords: friction stir processing, stirring, nanostructuring, thermomechanically affected zone.

\section{INTRODUCTION}

Friction stir processing is now a fast developing technique for subsurface structural modification of different materials including not only aluminum alloys but steels and alloys [1]. One of the ways is using the friction stir processing for introducing the reinforcing hard particles into the subsurface layers. Another one is the friction stir processing for refining the grain structure. The friction stir processing is a promising technique for obtaining hardened layers on the machine components and in this sense it may be analogous to the nanostructuring burnishing when the subsurface of a steel sample is hardened to the depth several microns below the surface, but such a processing technique requires setting up the process parameter values to the high accuracy. In case of friction stir processing, the thickness of a modified layer is determined only by the geometry and strength of the friction stir processing tool.

The effect of friction stir processing hardening by the grain structure refining has been observed when hardness increased to $1000 \mathrm{HV}$ [2]. Also friction stir processing of high-carbon steel has been carried out to determine the wear resistance of the processed layer which was one order of magnitude higher than that of non-processed metal [3]. The effect of hardening was related to quenching and martensite transformation of the friction stir processed grains. The same effect has been described elsewhere [4] for FSW of $0.72 \% \mathrm{C}$ steel when it was possible to obtain either pearlite or martensite structure controlling the cooling rate and temperature during FSW.

A good progress has been achieved recently in studying the nanosized microstructures produced by severe plastic deformation in metals including those produced by sliding $[5,6]$. In our opinion, these achievements have to be taken into account when studying the microstructural modification by friction stir processing. In this connection more research effort should be focused on friction stir processing in different materials including the high-melting point ones.

Summarizing the literature review, we may conclude that friction stir processing is a promising severe plastic deformation technique for research and development purposes.

The objective of this work is to study the friction stir processing for hardening and wear of the medium carbon steel.

International Conference on Physical Mesomechanics of Multilevel Systems 2014

AIP Conf. Proc. 1623, 627-630 (2014); doi: 10.1063/1.4899023

(C) 2014 AIP Publishing LLC 978-0-7354-1260-6/\$30.00 


\section{MATERIAL AND RESEARCH}

The friction stir processing of medium carbon steel samples has been carried out using a milling machine operated at $1500 \mathrm{rpm}$ and feed rate $23 \mathrm{~mm} / \mathrm{min}$. The friction stir processing tools have been machined from tungsten carbide-base hard metals of $8 \mathrm{wt} . \%$ and $20 \mathrm{wt} . \%$ cobalt content. The tools were shaped as (1) $2.5 \times 2.5 \times 1.5 \mathrm{~mm}$ and (2) $5 \times 5 \times 1.5 \mathrm{~mm}$ tetrahedrons and had the shoulder/pin size ratio 2.8 and 3.7 , respectively.

Wear resistance tests have been performed using a TriboTechnic tribometer and a reciprocal test scheme with an indentor made of $\mathrm{WC} / \mathrm{Co}$ hard metal. Test conditions were as follows: normal load $12 \mathrm{~N}$ for non-processed metal and $47 \mathrm{~N}$ for the processed metal under boundary lubrication by DC 705 vacuum oil. The sliding speed was 5 and $10 \mathrm{~mm} / \mathrm{c}$, respectively. The 3.7 shoulder/pin ratio tool processed metal was compared to the non-processed metal after grinding the samples to the depth of $0.2 \mathrm{~mm}$ to remove flash and large irregularities. The resulting wear scars have been analyzed using laser 3D profilometer New View Zygo.

\section{RESULTS \\ Microstructure}

The optical images transversal with respect to the tool feed direction show the subsurface layer obtained. We can distinguish several zones $A, B, C$ in the modified metal being stirred metal zone, heat affected zone and unchanged base metal, respectively.

For tool 1 a boundary between the processed layer and underlying base metal is thin and textured layers can be found being separated from each other by the "kissing bond"-type boundaries. For tool 2 one can see a dark zone $D$ layer on the top, then "white" non-etched sublayers of the $A$-zone and needle-like layered structures between the sublayers. The stirred zone ( $A$-zone) structure made by tool 1 may be characterized as a fine-grain and homogeneous without texturing, i.e. recrystallized with the mean grain size $2.3 \mu \mathrm{m}$ (zone $A$ in Fig. 1(a)). This microstructure is different from the inhomogeneous layered one obtained using tool 2, which is composed of light- and dark-etched sublayers (Fig. 1(b)). The interior microstructure of the $A$-zone white layers is not metallographically detectable and, as follows from the relevant literature sources, it may be presented by the fine-grain martensite. The white nonetched layers are common findings in wear testing of carbon steels [9]. The needle-like structures in the dark sublayers of the $A$ zone have their mean size about $30 \mu \mathrm{m}$ and could be either bainite or, what is more plausible, troostite structures.

The microhardness numbers profiles are in agreement with the results of microstructural characterization (Fig. 2). For friction stir processing by tool 1 we can see a thin layer of high hardness at the sample's surface (Fig. 2, curve 1 ). The underlying $A$-zone metal shows much lower hardness which becomes equal to that of the $C$-zone base metal at the depth $1.380 \mathrm{~mm}$ below the surface.

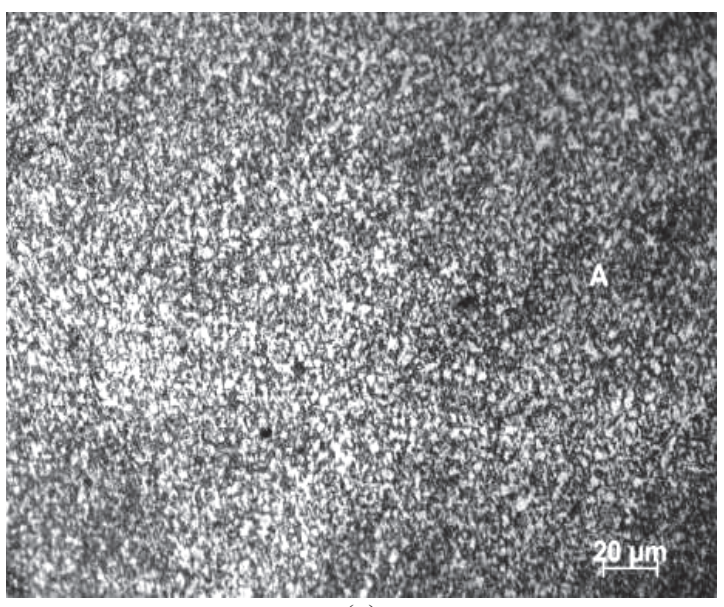

(a)

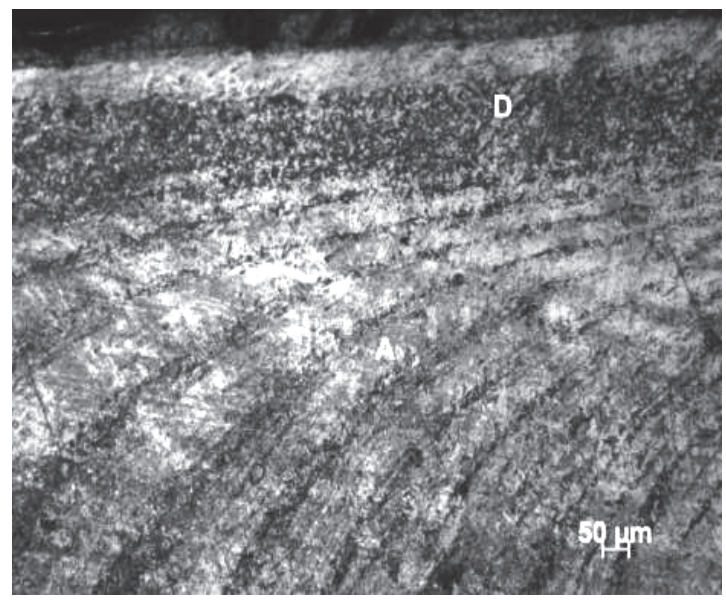

(b)

FIGURE 1. The microstructure of stirred zone metal for tool 1 (a) and 2 (b). $A$ - stirred zone, $D$ - shoulder-driven metal zone 


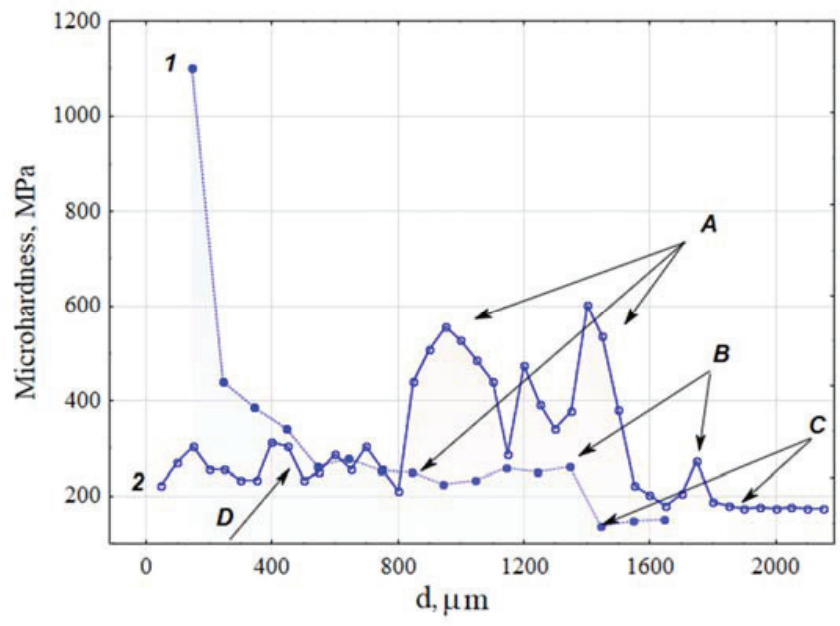

FIGURE 2. The microhardness numbers distribution for tool 1 (curve 1 ) and 2 (curve 2) below the processed metal surface

3D interferometry wear scar image of non-processed metal is $0.4 \mathrm{~mm}$ width with a deep groove (Fig. 3(a)). The distribution of microhardness numbers for FSW by tool 2 in $A$-zone is also inhomogeneous. Comparing the microstructure and microhardness data we can see that white non-etched sublayers possess higher 500-600 MPa hardness as compared to the dark ones with hardness at the level of 300-350 MPa. Another structural difference between the samples is the presence of zone $D$ with hardness $250-300 \mathrm{MPa}$ at the top of the tool 2-processed sample. This zone is formed by the shoulder-driven metal in contrast to the pin-driven metal of the $A$-zone.

\section{Wear}

3D interferometry wear scar image of non-processed metal is $0.4 \mathrm{~mm}$ width with a deep groove (Fig. 3(a)). The wear scar of friction stir processed sample is only slightly visible on the background of the rest of the surface area in spite of the fact that the wear path length was six times as long as that of the non-processed sample, i.e. $120 \mathrm{~m}$ against $20 \mathrm{~m}$. Therefore, we have carried out new testing at $47 \mathrm{~N}$ load and $200 \mathrm{~m}$ wear path length (Fig. 3(b)) which allowed us to measure the wear scar width more accurately. It is $0.23 \mathrm{~mm}$. The wear intensity of non-processed and processed samples was 0.02 and $0.001 \mathrm{~mm} / \mathrm{m}$, respectively.

\section{DISCUSSION}

Generation of the layered structure can find its explanation basing upon the results reported [6, 7] where the influence of strain inhomogeneities on generation of layered structure in sliding has been discussed from the viewpoint of shear instability.

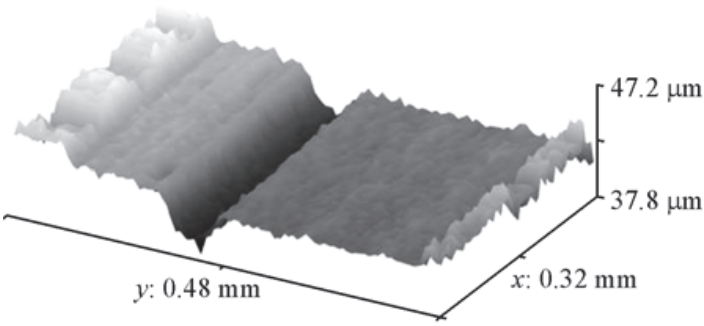

(a)

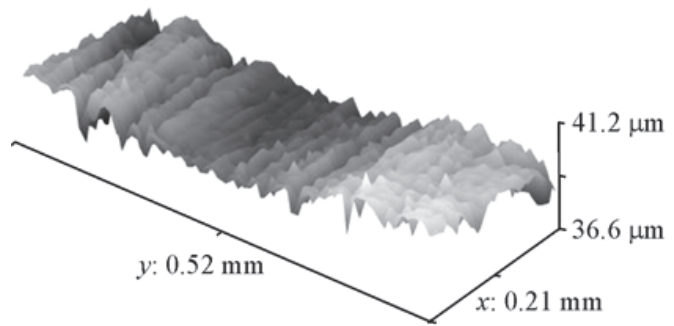

(b)

FIGURE 3. The worn surface topology for samples processes by tool 2 :

(a) sample 1-3, $12 \mathrm{~N}, 20 \mathrm{~m}, 5 \mathrm{~mm} / \mathrm{s}$; (b) sample 2-3, $47 \mathrm{~N}, 200 \mathrm{~m}, 10 \mathrm{~mm} / \mathrm{s}$ 
It was shown that severe plastic deformation in sliding may induce the subsurface structural modification which then results in quasi-viscous flow of the subsurface sublayers with respect to the underlying metal and each other. Also, in contrast to the viscous fluid dynamics the sublayers flow demonstrates its specificity stemming from the feasibility of shearing between the neighboring sublayers. So, the most intense plastic strain is localized in sublayers, whereas there may be only the elastic strain between them.

Taking this into account, we may explain the presence of the bainite (troostite) structures at the boundaries between the white non-etched layers after friction stir processing. The adhesion of plasticized metal creates sliding condition being the same as that observed in the course of adhesive wear on ductile metals [7]. Such a situation is characterized by the presence of tribological layer on the surface of metals in contact. This tribological layer serves as an intermediate material, which sticks both to the tool and the processed metal. Under this condition the stirring efficiency of the FSW tool is reduced and the sublayer flow pattern of plasticized metal driven by the tool is established as discussed [6-8].

According to [9], bainitic reaction occurs due to carbon content dependence of the martensitic transformation onset and by redistribution of carbon under isothermal conditions in the temperature range above this temperature. Also, the temperature of martensitic transformation onset is lower for the fine-grain medium carbon steels. All these factors induce the bainitic reaction under the extra heat release in the sublayers in stirred zone after stopping the test.

It is also supported by the microhardness numbers (Fig. 2, zone $A$ ) where we can see peaks and valleys corresponding to the white and dark sublayers in Fig. 1.

The 3.7 shoulder/pin size ratio tool produced quenched structure while the 2.8-one produced recrystallized structures. This may be related to higher temperature achieved in the first case due to large area of plasticized metal swept by the shoulder.

The stirred zone diameter is limited by the pin-driven part of stirred metal which may be affected by the adhesion of metal to the FSW tool surface.

\section{CONCLUSION}

As shown, the use of FSW tool with larger shoulder area has resulted in forming the processed seam layered structure composed of hard nanocrystalline quenched non-etching sublayers and bainite crystals at the sublayer boundaries. The use of the smaller FSW tool has resulted in generation of submicron size recrystallized grains in the stirred zone and layered but not quenched structure of thermomechanically affected zone.

The wear resistance of the quenched layered structures is an order of magnitude higher than that of nonprocessed medium carbon steel.

\section{ACKNOWLEDGEMENT}

This work has been supported by RFBR (grant No. 13-08-98088).

\section{REFERENCES}

1. E. D. Nicholas, Weld. World 47(11-12), 2 (2003).

2. S. Kuichi and H. Sasahara, J. Adv. Mech. Design Syst. Manufact. 4(5), 838 (2010).

3. S. H. Aldajaha, O. O. Ajayi, G. R. Fenske, and S. David, Wear 267, 350 (2009).

4. L. Cui, H. Fujii, N. Tsuji, and K. Nogi, Scripta Mater. 56, 637 (2007).

5. S. Yu. Tarassov and A. V. Kolubaev, Wear 231, 228 (1999).

6. S. Tarasov, V. Rubtsov, and A. Kolubaev, Wear 268, 59 (2010).

7. V. E. Rubtsov, S. Yu. Tarasov, and A. V. Kolubaev, Phys. Mesomech. 15(5-6), 337 (2012).

8. S. Yu. Tarasov and V. E. Rubtsov, Phys. Sol. State 53(2), 358 (2011).

9. A. P. Gulyaev, The Metal Science (Metallurgy, Moscow, 1986). 